\title{
INVESTIGAÇÃO DO MODO SUL EM DADOS DE PRECIPITAÇÃO NO PERÍODO DE 1982 A 2006 NO ESTADO DO RIO GRANDE DO SUL
}

\author{
LUANA ALBERTANI PAMPUCH, SIMONE EROTILDES TELEGINSKI FERRAZ
}

\author{
Universidade Federal de Santa Maria, Santa Maria, RS, Brasil \\ lupampuch@gmail.com,simonetfe@ufsm.br
}

Recebido Setembro de 2010 - Aceito Setembro de 2011

\begin{abstract}
RESUMO
Este trabalho apresenta o estudo de um modo de variabilidade que influencia a precipitação no Sul do Brasil e é chamado Modo Sul de precipitação. Será mostrado que a ocorrência de máximos (e mínimos) do Modo Sul de precipitação pode estar relacionada à ocorrência de eventos extremos no Rio Grande do Sul, como vendavais, enchentes, granizo e estiagens

Utilizando a análise de componentes principais em dados de precipitação diária filtrados na banda 10-50 dias, são encontrados campos espaciais e temporais que representam a máxima variância de determinadas variabilidades, e neles são detectados Modos de Variabilidade de precipitação. Desta maneira, foi possível determinar este modo de variabilidade, que aparece bem configurado na região do Rio Grande do Sul. A série de componentes principais foi usada para a escolha desses eventos. O Modo Sul foi calculado para o período de 01/03/1982 à 31/05/2006. Foi possível identificar que os eventos extremos chuvosos ocorreram em maior número do que os eventos extremos secos. $\mathrm{Na}$ análise decadal verificou-se um aumento no número de eventos, quando comparada às décadas de 80,90 e 2000. Vale ressaltar que o número de eventos da década de 2000 em relação à década de 80 (proporcionalmente) foi o mais expressivo.
\end{abstract}

Palavras-Chave: Climatologia, Modo Sul de precipitação, desastres naturais.

\begin{abstract}
RESEARCH OF "MODO SUL" ON RAINFALL DATA DURING THE PERIOD FROM 1982 TO 2006 IN RIO GRANDE DO SUL STATE

This work presents a study of a rainfall variability mode that acts in southern Brazil and is called "Modo Sul" of precipitation. It was tried to show that the occurrences of maximum (and minimum) of the "Modo Sul" of precipitation are related to the occurrence of extreme events in Rio Grande do Sul, as windstorm, flood, hail and drought.

Using principal component analysis of daily filtered precipitation data for the 10-50 day band we found spatial pattern and temporal series that represent the maximum variance of certain variabilities, which are the modes of precipitation variability. Thus, it was possible to determine the mode of variability that appears well established in Rio Grande do Sul. The principal component series was used to select the extreme events.

The "Modo Sul" was calculated for the period from 01/03/1982 to 31/05/2006. It was possible to realize that the extreme rainy events were more frequent than extreme dry events. In a decadal analysis an increase in the number of events was found when comparing the $80 \mathrm{~s}, 90 \mathrm{~s}$ and 2000 . It is noteworthy that the number of events of 2000s compared with the 80 s was proportionally the most expressive.
\end{abstract} Keywords: Climatology, "Modo Sul" of rainfall, natural disasters. 


\section{INTRODUÇÃO}

Eventos extremos como enchentes, vendavais e granizo são processos da dinâmica superficial e atmosférica. Estes ocorrem independentemente da presença do homem, e são manifestações próprias da natureza. Porém, quando esses processos se deslocam sobre áreas habitadas, geram situações de perigo a pessoas e bens, podendo acarretar grandes impactos econômicos e sociais. Em geral, as áreas urbanas não são adequadamente projetadas para absorver grandes quantidades de chuva, assim, eventos extremos de precipitação, causam grandes transtornos à população. Além disso, períodos prolongados de seca podem causar abaixamento nos níveis dos reservatórios, causando desabastecimento nos centros urbanos e na produção de energia hidroelétrica.

A região sul do Brasil, além de amplamente povoada, possui uma economia basicamente agrícola, sendo desta forma, altamente dependente da precipitação. Portanto, conhecer a variabilidade da precipitação e os mecanismos atmosféricos, que produzem extremos secos e chuvosos, torna-se indispensável. Pois culturas agrícolas, também, são altamente influenciadas pelas condições de tempo (Streck et. al., 2008) e a água é fundamental no metabolismo das plantas e uma redução na sua disponibilidade no solo (ou um aumento desproporcional) pode afetar o crescimento, o desenvolvimento e a produtividade das culturas (Sinclair e Ludlow, 1986).

A precipitação do Sul do Brasil é afetada por diversos fenômenos de variabilidade interanual, como o El Niño, e de baixíssima freqüência, como a Oscilação Decadal do Pacífico (ODP), passando também por fenômenos da escala intrasazonal, como a Oscilação de Madden-Julian (OMJ - Madden e Julian, 1971, 1994).

Diversos autores tentaram caracterizar fenômenos e modos de variabilidade que influenciam a precipitação na América do sul. Ao caracterizar as variações anuais da chuva sobre o Brasil, Rao e Hada (1990) analisaram a relação entre precipitação no Brasil e o IOS (Índice de Oscilação Sul) e verificaram que o coeficiente de correlação, entre o IOS e a chuva, é geralmente pequeno sobre quase todo o Brasil com exceção do estado do Rio Grande do Sul (RS). A correlação da chuva na primavera com o IOS na mesma estação ou na anterior é significativamente alta e mostra grandes perspectivas para previsões de chuvas sazonais. Os autores sugeriram que na fase negativa da Oscilação Sul ocorre um fortalecimento da circulação de Hadley, que por sua vez transporta mais momento angular absoluto, mantendo um jato subtropical mais forte. Esta situação do escoamento em altitude mantém os sistemas frontais no sul do Brasil, favorecendo o maior acúmulo de chuva nesta região e reduzindo a precipitação no Nordeste Brasileiro (NEB). Fernandes et. al. (2009) analisaram especificamente a relação entre o IOS e a precipitação no RS. Foram encontradas correlações significativas entre as precipitações de primavera no RS e o IOS, e estas são predominantemente negativas. O IOS apresentou correlações mais significativas com as precipitações no RS no bimestre de outubro-novembro, o que já havia sido observado por Rao e Hada (1990). No bimestre novembrodezembro apenas as regiões central e oeste apresentaram correlações significativas. Nos bimestres dezembro-janeiro e janeiro-fevereiro, as correlações entre as precipitações e o IOS não apresentaram significância estatística.

A ODP é uma flutuação da temperatura da superfície do mar no Pacífico em uma escala de tempo interdecadal. A região tropical do Oceano Pacífico e a costa do continente norte americano se tornam mais frias (quentes), enquanto sua região extratropical se torna mais quente (fria). Mantua et al. (1997) descreveram a ODP como sendo um evento El Niño - Oscilação Sul de longa duração e da mesma forma que o ENOS, a ODP apresenta duas fases. A fase fria é caracterizada por anomalias negativas de TSM no Oceano Pacífico Tropical e, simultaneamente, anomalias de TSM positivas no Oceano Pacífico Extratropical em ambos os hemisférios. A última fase fria ocorreu no período 1947-1976. Já a fase quente apresenta configuração contrária, com anomalias de TSM positivas no Oceano Pacífico Tropical e negativas no Oceano Pacífico Extratropical. A fase quente se estendeu de 1977 a 1998. Atualmente a configuração oceânica apresenta sinais de uma nova fase fria da ODP. O índice da ODP (IODP) foi desenvolvido por Hare (1996) e Zhang (1996) e é definido como a diferença entre as anomalias observadas e a média mensal global da anomalia da TSM (Zhang et al., 1997). Rebello (2006) analisou a possível influência da ODP na precipitação do RS relacionando o IODP com a precipitação média no RS. Seus resultados mostraram que durante a fase fria da oscilação foram observadas anomalias negativas de precipitação no RS, e durante a fase quente da oscilação foram observadas anomalias positivas de precipitação nesse estado. O mesmo foi observado por Cera et. al. (2009), que verificou que a correlação entre anomalias de precipitação e o IODP é mais significativa no Nordeste do estado do RS, exceto no verão em que a região preferencial é a central.

$\mathrm{Na}$ América do Sul, fenômenos da escala intrasazonal também atuam de forma a modular o clima da região. Um exemplo disto é a OMJ, que causa variações na posição e intensidade da Zona de Convergência do Atlântico Sul (ZCAS), que por sua vez, interfere na precipitação das Regiões Centrooeste, Sudeste, setor sul do Nordeste e norte da Região Sul. Ainda dentro desta escala, podem-se destacar outros modos de variabilidade de precipitação determinados em Ferraz (2000, 2004). Estes modos foram denominados "Modo Nordeste- Sul (NES)", "Modo ZCAS - Norte (ZCN)", "Modo ZCAS - Sul (ZCS)" e "Modo SUL (SUL)". 
Outro modo de variabilidade de precipitação bem conhecido nesta escala foi determinado por Paegle e Mo (1997) e Paegle et. al. (2000), que representa alternância de condições secas e úmidas sobre a América do Sul tropical e Subtropical. Kidson (1999) também estudou os principais modos de variabilidade de baixa frequência da circulação atmosférica através da função corrente em $300 \mathrm{hPa}$ no Hemisfério Sul, e seus resultados mostraram que os modos preferenciais de variação são estacionários ou são trens de onda de número de onda 4 ou 5 , que se propagam para leste e estão confinados em médias e altas latitudes.

Tendo em vista os resultados encontrados em Ferraz (2004) e baseado nos trabalhos de outros autores, o objetivo deste trabalho é analisar a relação do modo de variabilidade de precipitação denominado "Modo Sul", com eventos extremos de precipitação ocorridos no sul do Brasil para o período de 01/03/1982 á 31/05/2006. Ferraz (2004) calculou o Modo Sul apenas para o período de novembro à março durante os anos de 1965 á 1990, pois o enfoque principal daquele trabalho era verificar a estação chuvosa do Sudeste do Brasil. Como o Rio Grande do Sul não apresenta uma estação chuvosa bem definida, apresentando-se com altos índices pluviométricos distribuídos durante todo o ano (Cera e Ferraz, 2007) o novo cálculo do Modo Sul foi realizado para o ano inteiro (janeiro à dezembro). Com isso foi possível verificar qual estação (verão, outono, inverno ou primavera) se apresenta mais seca ou mais chuvosa, e o período de março de 1982 à maio de 2006 foi escolhido para caracterizar o Modo em um período mais recente.

Conforme foi determinado em Ferraz (2004), o Modo Sul aparece em todas as subbandas intrasazonais de variabilidade, sendo caracterizado por um núcleo na região entre $25^{\circ} \mathrm{S}$ e $35^{\circ} \mathrm{S}$ de latitude e $52^{\circ} \mathrm{W}$ e $62^{\circ} \mathrm{W}$ de longitude. A configuração espacial do Modo Sul será apresentada na sessão 3.

\section{MATERIAL E MÉTODOS}

Foram utilizados dados diários de precipitação para o período de $1^{\circ}$ de março de 1982 à 31 de maio de 2006 de um conjunto de dados compilado e gentilmente disponibilizado pelo Dr. Brant Liebmann (NOAA - CIRES Climate Diagnostics Center - Liebmann e Allured, 2005), e disponibilizados no website: http://www.esrl.noaa.gov/psd/people/brant.liebmann/ south_america_precip.html. Este conjunto é formado por dados diários de diversas fontes de pluviômetros na América do Sul, possui uma boa cobertura espacial e estão dispostos em pontos de grade de $1^{\circ}$. Para facilitar a análise, cada ponto de grade de $1^{\circ}$ foi considerado como representativo de uma única estação, e a partir deste ponto será denominada 'estação'.

Foi realizada uma seleção das estações com maior cobertura temporal de dados, sendo usadas apenas estações com no mínimo $80 \%$ dos dados (estações com uma quantidade maior de dados faltantes poderiam comprometer a confiabilidade dos resultados). De todas as estações usadas, 258 possuem pelo menos $80 \%$ dos dados. A distribuição destas estações é mostrada na Figura 1.

Após a seleção das estações, foram calculadas as climatologias diárias de cada estação para que estas pudessem ser usadas no preenchimento de dados faltantes. Com as estações preenchidas, a séries foram filtradas usando o Filtro de Lanczos (Duchon, 1979). Foi escolhida a banda 10/50 dias baseado nos resultados de Kidson (1999) e em experimentos preliminares mostrados em Ferraz (2004).

O Modo Sul de precipitação foi calculado utilizando a análise de Componentes Principais (ACP) com rotação e sem rotação (Lorenz, 1956 e Wilks, 1995).

$\mathrm{Na}$ análise sem rotação segundo Ferraz (2004), a orientação do primeiro autovetor no espaço K-dimensional é determinada pela direção da máxima orientação dos dados e os autovetores subsequentes devem ser ortogonais aos previamente determinados sem considerar os processos físicos envolvidos. No entanto, Cardoso (2005) alerta que embora sejam bons descritores da variabilidade de um conjunto de dados, estes modos (produzidos via $\mathrm{ACP}$ ) não necessariamente conduzem às interpretações claras dos processos físicos envolvidos no conjunto original, já que a influência de diferentes processos físicos pode estar contida em um só modo. Como os diferentes modos de variabilidade no mundo real não precisam ser ortogonais no espaço e no tempo, a rotação dos componentes principais pode ajudar na interpretação física destes modos.

Neste trabalho optou-se por utilizar a rotação do tipo Varimax, que é aceita como a rotação ortogonal algebricamente mais acurada, quando aplicada a uma série de dados "conhecidos" (Rummel, 1970; Mulaik, 1972; Harman, 1976). Para selecionar os modos que seriam rotacionados utilizou-se a regra de Kaiser (Kaiser 1958, 1959), que consiste em selecionar o número de modos que somam mais do que a quantidade média da variância total do conjunto original (variância amostral):

$$
\lambda_{\mathrm{n}}=\frac{\mathrm{T}}{\mathrm{K}} \sum_{\mathrm{k}=}^{\mathrm{K}} \mathrm{s}_{\mathrm{k}, \mathrm{k}}
$$

sendo $s_{\mathrm{k}, \mathrm{k}}$ a variância do $\mathrm{k}_{\mathrm{th}}$ elemento e $\mathrm{T}$ um parâmetro deste limiar que varia de 0,7 a 1 . Neste trabalho utilizamos $\mathrm{T}=0,7$, conforme sugerido por Jolliffe (1972). Esse método é mais utilizado quando se tem uma grande quantidade de elementos.

Paegle e Mo (1997) haviam utilizado esta mesma técnica aplicada a dados de radiação de onda longa (ROL), filtrados na banda 10-90 dias. Eles determinaram os eventos extremos a partir do desvio padrão dos componentes principais (CP). Eventos positivos (negativos) foram escolhidos quando o $\mathrm{CP}$ era maior (menor) do que um determinado limiar por mais 


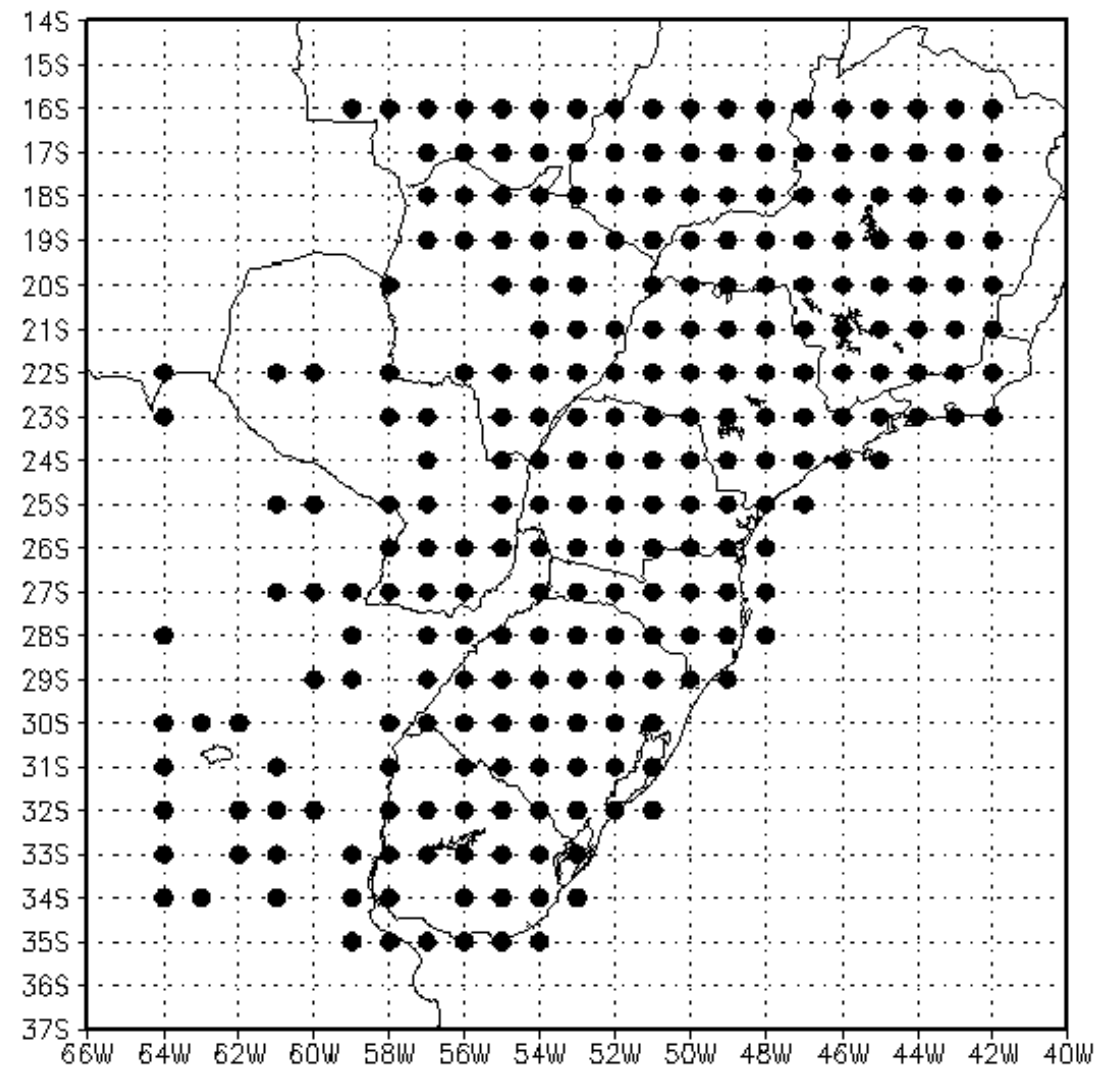

Figura 1- Estações utilizadas a partir dos dados de precipitação de Liebmann e Allured (2005).

de cinco (5) dias consecutivos (o limiar utilizado foi de $\pm 1,2$ vezes o próprio desvio padrão). No presente trabalho, o limiar considerado foi de $\pm 1,35$ vezes o desvio padrão, para restringir eventos mais extremos. Além disso, foram considerados apenas os eventos encontrados na série de $\mathrm{CP}$ com rotação e que tivessem o CP excedendo o valor limite por no mínimo três (3) dias consecutivos. Neste trabalho o sinal negativo do Modo Sul sobre o RS está relacionado com anomalias positivas de precipitação como será visto na sessão 3.

As datas do Modo Sul encontradas utilizando ACP foram comparadas com datas de desastres naturais ocorridos no Rio Grande do Sul. Reckziegel (2007) realizou um levantamento dos Desastres Naturais no Estado no período de 1980 a 2005, utilizando dados do Diário Oficial, Defesa Civil do Rio Grande do Sul e dados do Jornal Zero Hora. Os eventos de estiagens e secas foram classificados como eventos extremos secos e os eventos de vendaval, vendaval e granizo, enxurrada, enchente, granizo e tornado foram classificados como eventos extremos chuvosos. Estes dados foram usados para validar as datas do Modo Sul da seguinte forma: o evento do Modo Sul foi considerado como confirmado no caso em que pelo menos um município do Rio Grande do Sul se apresentou na lista de Reckziegel (2007) no período de mais (menos) três dias após (antes) a data encontrada na ACP. Para o ano de 2006, a mesma técnica utilizada por Reckziegel (2007) foi utilizada para fazer um levantamento dos eventos ocorridos neste ano, utilizando dados dos jornais Correio do Povo, A Razão e Diário de Santa Maria e Defesa Civil do RS.

Foram avaliadas, também, a ocorrência do Modo Sul por ano, estação do ano e realizada uma análise decadal, a fim de verificar a importância deste Modo em cada década. Uma análise comparativa (número de eventos, quantidade de extremos, etc.) foi feita para quantificar se ocorreram mudanças nestes valores.

\section{RESULTADOS E DISCUSSÃO}

O Modo Sul foi encontrado como quarto modo de variabilidade na análise com rotação e explicou 7,75\% da variância total (Figura 2). Os três primeiros modos (não mostrados) explicam juntos 35,86\% da variância total. Estes três modos correspondem aos modos ZCAS, ZCS e NES encontrados em Ferraz (2004). Como neste trabalho, o interesse se restringe a relação entre o Modo Sul e a precipitação do RS, estes outros modos não foram considerados. No entanto, Ferraz (2004) já havia mostrado que estes três modos, mais o Modo Sul, são independentes entre si e representam processos físicos distintos.

$\mathrm{Na}$ Figura 2 são plotados os coeficientes espaciais da análise de componentes principais,em que são observados 
valores negativos na região sul do Brasil e positivos no sudeste; sendo que no sul estes valores são superiores em módulo do que na região sudeste. Estas evidências caracterizam o Modo Sul (Ferraz, 2000, 2004). Para se determinar eventos extremos secos e chuvosos tem-se que analisar a série de $\mathrm{CP}$, juntamente com dados reais de precipitação do período escolhido (figuras não mostradas). Neste caso, valores positivos da $\mathrm{CP}$ correspondiam a déficit de precipitação e valores negativos da $\mathrm{CP}$ a extremos positivos de precipitação, e as regiões em que estes valores extremos ocorriam coincidem com o máximo sinal do Modo de variabilidade (Figura 2). Assim, valores negativos no modo representavam eventos chuvosos do Modo Sul, enquanto que valores positivos representavam eventos secos (Pampuch, 2010).

Foram encontrados 135 eventos chuvosos e 108 eventos secos do Modo Sul. Com os valores dos desvios padrões $(\sigma)$, é possível fazer uma análise da intensidade dos eventos do Modo Sul conforme descrito na Tabela 1. Valores, em módulo, muito acima do limiar considerado $(1,35)$ representam eventos muito extremos.

Foram destacados o mínimo e máximo desvio padrão, e o valor médio foi calculado (média de todos os valores), tanto dos eventos secos, quanto chuvosos (Tabela 2). É possível observar que tanto nos eventos extremos secos, quanto nos chuvosos os valores médio e máximo ficaram acima do valor considerado como limiar $(1,35)$. Além disso, os eventos extremos chuvosos foram mais intensos do que os secos, pois apresentaram, em módulo, valores mais intensos para máximo desvio padrão e desvio padrão médio.

A duração dos eventos secos e chuvosos do Modo Sul pode ser vista na Figura 3. Foram considerados apenas os eventos encontrados na série de $\mathrm{CP}$ com rotação e que tiveram o CP excedendo o valor limite $(1,35)$ por no mínimo 3 dias consecutivos. Os eventos secos tiveram duração entre 3 e 7 dias, sendo que os eventos de 4 dias ocorreram com maior frequência. Os eventos chuvosos tiveram duração entre 3 e 9 dias, sendo que os eventos de 3 e de 6 dias ocorreram com maior freqüência . Desta forma, os eventos secos se apresentaram mais curtos do que os chuvosos.

O ano de 2002 foi o que apresentou maior ocorrência de eventos de extremos secos do Modo Sul (12 eventos), enquanto que nos anos de 1989, 1996 e 2006 não houve registro de ocorrência destes eventos. Já para os eventos chuvosos, tanto o ano de 1983, como o de 2002, foram os que apresentaram o maior número de eventos, um total de 9 em cada ano. Já o ano de 2006 não apresentou nenhum evento chuvoso (Figura 4). Um destaque para o ano de 2002 que apresentou um número elevado de eventos, tanto secos, quanto chuvosos, este ano foi caracterizado por evento de El Niño moderado que pode ter modulado a variabilidade intrasazonal.

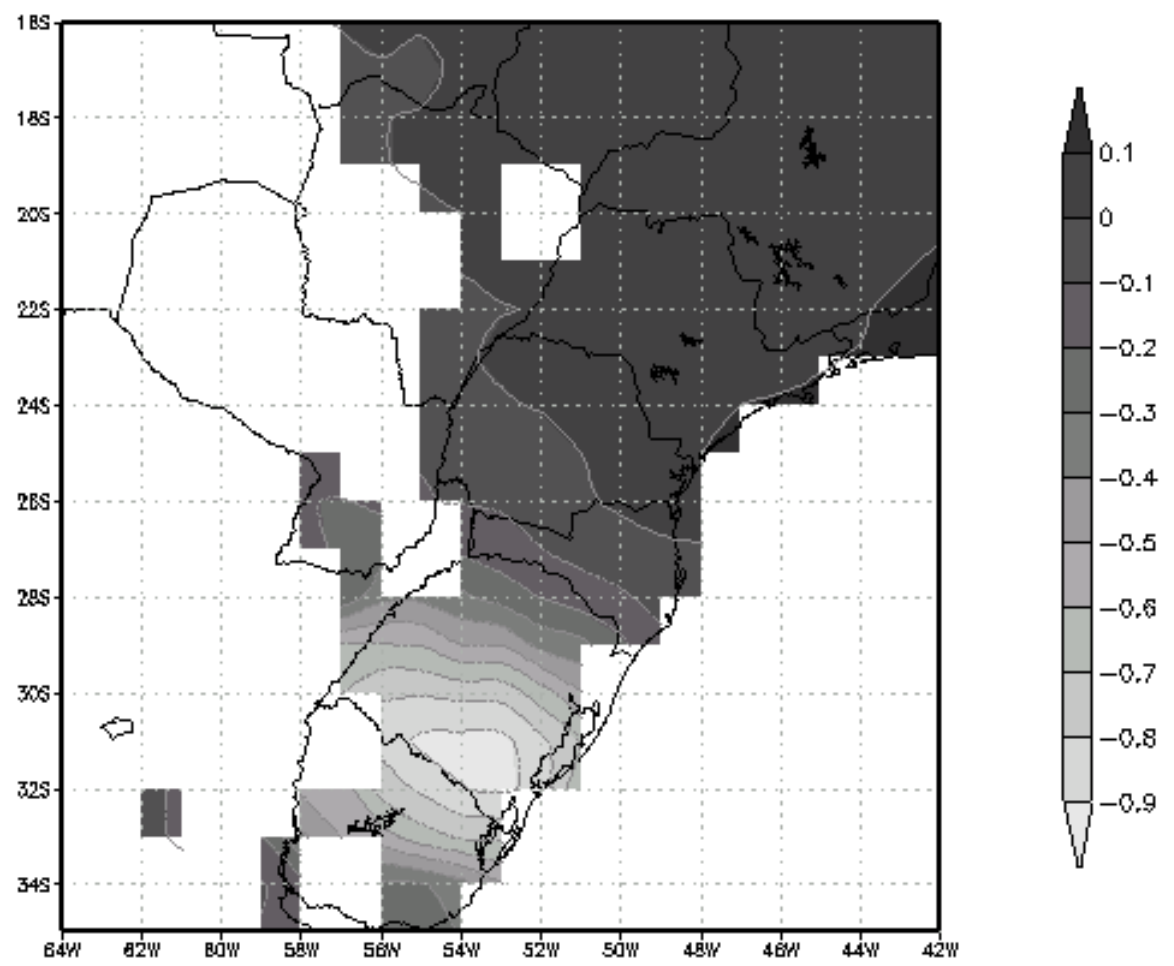

Figura 2- Padrão espacial da $4^{\circ}$ componente principal rotacionada de dados filtrados de precipitação - denominado Modo Sul. 
Tabela 1 - Desvio padrão considerado na seleção dos eventos extremos.

\begin{tabular}{ll}
\hline Extremos chuvosos & $\sigma<-1,35$ \\
\hline Extremos secos & $\sigma>1,35$ \\
\hline Normal & $-1,35 \leq \sigma \leq 1,35$ \\
\hline
\end{tabular}

Tabela 2 - : Resumo dos resultados encontrados para os eventos do Modo Sul no período de 01/03/1982 à 31/12/1989.

\begin{tabular}{|c|c|c|c|c|c|c|c|c|}
\hline EVENTOS & $\begin{array}{c}\text { NÚMERO } \\
\text { DE } \\
\text { EVENTOS }\end{array}$ & $\begin{array}{l}\text { MÁXIMO } \\
\text { DESVIO } \\
\text { PADRÃo } \\
\text { (MÓDULO) }\end{array}$ & $\begin{array}{l}\text { MÍNIMO } \\
\text { DESVIO } \\
\text { PADRÃO } \\
\text { (MÓDULO) }\end{array}$ & $\begin{array}{c}\text { DESVIO } \\
\text { PADRÃO } \\
\text { MÉDIO } \\
\text { (MÓDULO) }\end{array}$ & $\begin{array}{c}\text { DURAÇÃO } \\
\text { DOS } \\
\text { EVENTOS } \\
\text { (DIAS) }\end{array}$ & $\begin{array}{c}\text { ANO COM } \\
\text { MAIOR } \\
\text { NÚMERO } \\
\text { DE } \\
\text { EVENTOS }\end{array}$ & $\begin{array}{c}\text { ESTAÇÃO } \\
\text { COM MAIOR } \\
\text { NÚMERO DE } \\
\text { EVENTOS }\end{array}$ & $\begin{array}{l}\text { ANÁLISE } \\
\text { DECADAL }\end{array}$ \\
\hline SECOS & 108 & 3,428 & 1,448 & 2,108 & 3 a 7 & 2002 & Outono & $\begin{array}{c}80-90= \\
+3,61 \% \\
90-2000= \\
+36,87 \% \\
80-2000= \\
+41,76 \%\end{array}$ \\
\hline CHUVOSOS & 135 & 6,1199 & 1,434 & 2,623 & 3 a 9 & $\begin{array}{c}1983 \text { e } \\
2002\end{array}$ & $\begin{array}{c}\text { Bem } \\
\text { distribuída }\end{array}$ & $\begin{array}{c}80-90= \\
+2,02 \% \\
90-2000= \\
+0,19 \% \\
80-2000= \\
+2,22 \%\end{array}$ \\
\hline
\end{tabular}

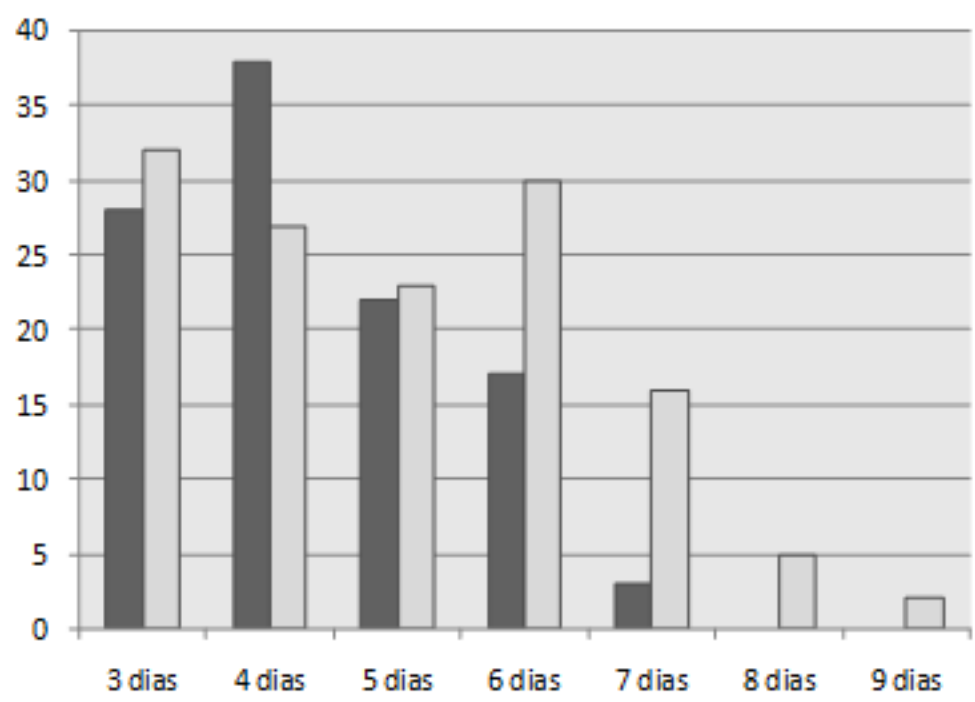

Figura 3 - Duração dos eventos secos (preto) e chuvosos (cinza) do Modo Sul no período de 01/03/1982 to 31/05/2006. 


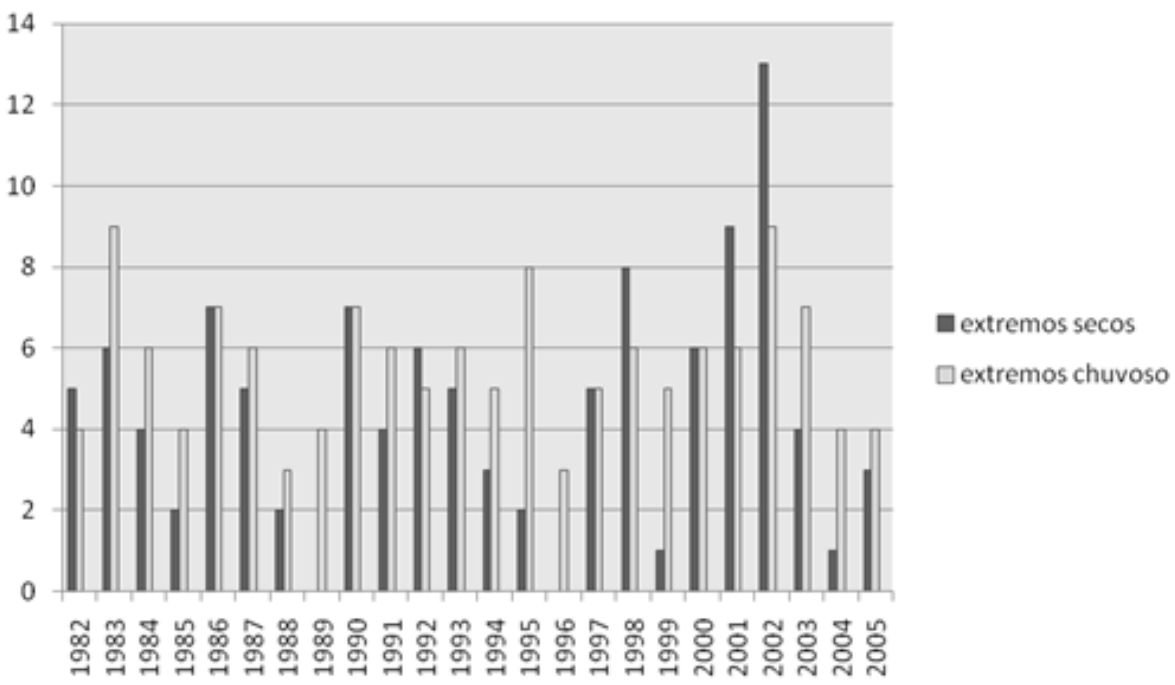

Figura 4 - Número dos eventos extremos secos (preto) e chuvosos (cinza) do Modo Sul por ano no período de 1982 a 2006.

A década de 80 (1982 -1989) é composta do período de 01/03/1982 à 31/12/1989, apresentando 7,8 anos. Nesta década foram encontrados 31 eventos secos e 43 eventos chuvosos do Modo Sul, tendo média de 3,974 eventos secos por ano e 5,513 eventos chuvosos por ano. A década de 90 (1990-1999) é a única completa, apresentando 10 anos. Foram identificados 41 eventos secos e 56 eventos chuvosos do Modo Sul, assim, a década de 90 apresentou uma média de 4,1 eventos secos por ano e 5,6 eventos chuvosos por ano. Já na década de 2000 (2000-2006) o período considerado é de $01 / 01 / 2000$ à $31 / 05 / 2006$, tendo 6,4 anos. Como apresentou 36 ocorrências de eventos, tanto secos, quanto chuvosos do Modo Sul, obteve média de 5,6 eventos secos e chuvosos por ano.

Comparando as décadas de 80 e de 2000 , houve um aumento de 1,65 eventos secos por ano e de 0,1219 eventos chuvosos por ano, o que corresponde a um aumento de eventos secos em $41,79 \%$ ao ano e de $2,22 \%$ para eventos chuvosos. Assim, pode-se sugerir que os eventos extremos secos aumentaram da década de 80 para de 2000 e a quantidade de eventos chuvosos permaneceu praticamente a mesma (Tabela 2).

Fazendo uma análise por estação do ano, e considerando verão (de $21 / 12$ à $19 / 03)$, outono (20/03 à 20/06), inverno (21/06 à 21/09) e primavera (22/09 à 20/12), pode-se verificar que o outono foi a estação que obteve maior ocorrência de eventos extremos secos do Modo Sul (47 eventos), enquanto que nas outras estações ocorreram quase os mesmos números de eventos (no verão foram 18 eventos, no inverno 23 eventos e na primavera 20 eventos). Já para o caso de eventos chuvosos, nota-se que o número de eventos foi bem distribuído nas estações, o que mostra que o Rio Grande do Sul não apresenta uma estação típica chuvosa (Cera e Ferraz, 2007). A Figura 5 apresenta essa distribuição por estação do ano.
As datas dos eventos do Modo Sul foram comparadas com as datas de Reckziegel (2007). Um evento do Modo Sul foi considerado como confirmado, se a data deste evento coincidisse com a data de um evento de desastre natural que afetou pelo menos uma cidade da lista de Reckziegel (2007). Verificou-se que dos 108 eventos secos do Modo Sul, foram confirmados apenas 9 eventos. Desta forma, foram confirmados $8,33 \%$ dos eventos de extremos seco. Este valor tão baixo é devido à dificuldade para encontrar dados que caracterizem eventos secos ou estiagens, muitas vezes não são notificados em órgãos de Defesa Civil, ao contrário do que ocorre normalmente com os eventos chuvosos. Nos eventos chuvosos foram considerados para comparação apenas os eventos associados a desastres atmosféricos da lista de Reckziegel (2007): vendaval, enxurrada, enchente, granizo, tornado e vendaval e granizo em conjunto. Dos 135 eventos chuvosos do Modo Sul, 109 foram confirmados, o que corresponde a $80,74 \%$ dos eventos. Desta forma, sugere-se que o Modo Sul é responsável por grande parte da variabilidade da precipitação do RS causando extremos chuvosos.

Foi possível notar que a maioria dos eventos afetou mais de um município do Rio Grande do Sul. O evento mais notado foi de enchente (489 cidades afetadas), seguido do vendaval (433 cidades afetadas). Tornado foi o tipo de evento confirmado apenas 3 vezes no período de 01/03/1982 to 31/05/2006, atingindo 12 municípios. No entanto, pode-se notar que esses eventos ocorreram depois da década de 90. Nascimento e Calvetti (2004) demonstraram que o RS é uma região favorável à ocorrência de tornados, pois é onde ocorrem o encontro de correntes úmidas e quentes da Amazônia e as secas e frias vindas da Argentina.

Na Figura 6 são mostrados os eventos chuvosos ocorridos por mês. Pode-se notar que as enchentes ocorreram 


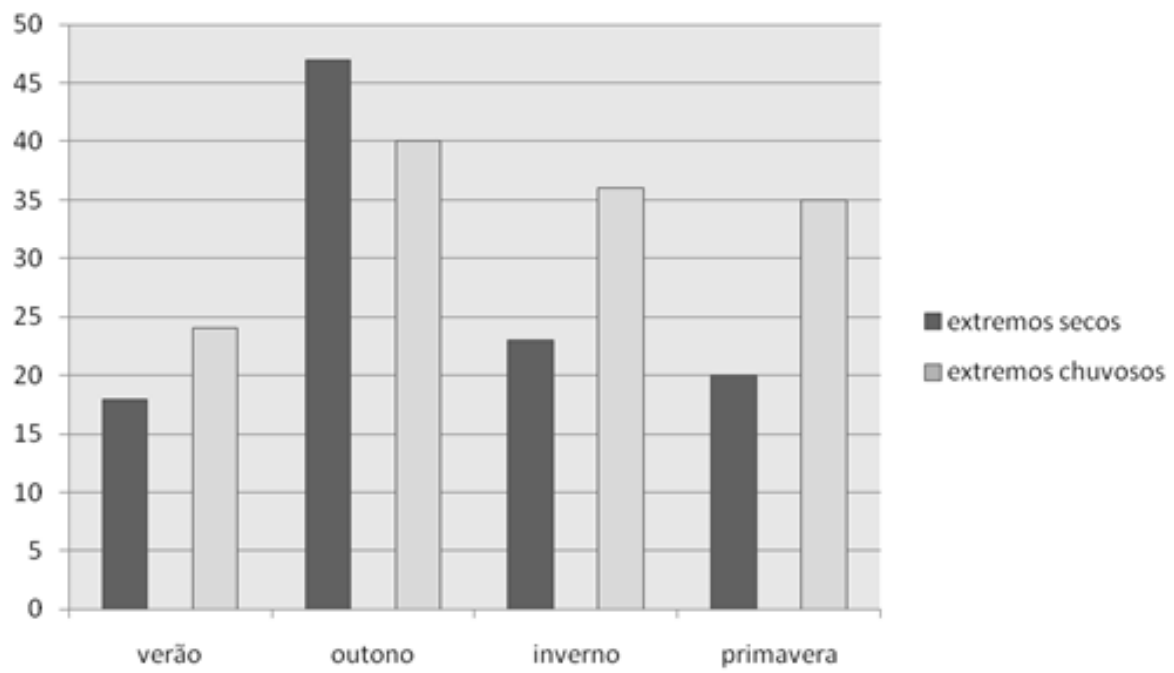

Figura 5 - Ocorrência dos eventos extremos secos (preto) e chuvosos (cinza) do Modo Sul por estação do ano de 1982 a 2006.

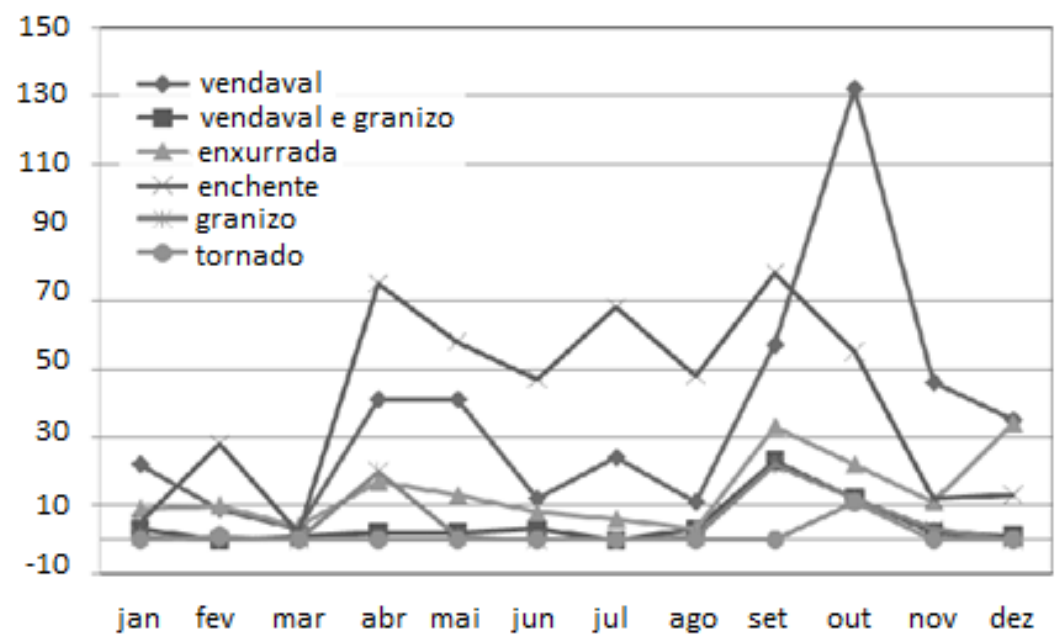

Figura 6 - Número de eventos extremos chuvosos do Modo Sul confirmados por Reckziegel(2007) por mês no período de 01/03/1982 to $31 / 05 / 2006$.

predominantemente nos meses de abril a setembro, sendo assim, mais características do final de outono e inverno e menos freqüentes no verão. Como já havia sido observado nos eventos chuvosos (Figura 5), o outono também apresentou maior número de eventos secos, o que é indicativo de uma estação de transição (períodos secos alternado com períodos chuvosos). Os eventos de vendaval tiveram um pico de ocorrência em outubro, os de granizo e tornado em setembro, e o de enxurrada de setembro a dezembro, sendo mais característicos da primavera. Este resultado reforça o fato de termos usado o período de janeiro a dezembro para o cálculo do Modo Sul.

\section{CONCLUSÕES}

Conhecer os modos de variabilidade que influenciam os extremos secos e chuvosos no Rio Grande do Sul é de grande importância, já que estes eventos trazem grandes prejuízos a população e a economia. O Modo Sul de precipitação é um desses modos.

Para o período considerado (01/03/1982 to 31/05/2006), foram encontrados 108 eventos secos e 135 eventos chuvosos do Modo Sul. Os extremos secos e chuvosos apresentaram valores bem acima de 1,35 desvios padrão considerado como limiar, o que pode ser um indicativo de eventos relativamente fortes. Os eventos secos se apresentaram mais curtos (4 dias) e os eventos chuvosos mais longos (6 dias), o Outono se apresentou como a estação de maior ocorrência de eventos secos e os eventos chuvosos ocorreram bem distribuídos durante o ano. Em uma análise decadal foi possível notar que a década de 2000 foi a que apresentou o maior número de eventos comparado as décadas de 80 e 90, o que sugere um aumento dos eventos do Modo Sul. 
Uma comparação com dados reais de desastres naturais mostrou uma grande concordância na data dos eventos chuvosos do Modo Sul, em que foram confirmados $80,74 \%$ dos eventos. Este fator mostra que o Modo Sul pode ser responsável por parte da ocorrência desses eventos no Rio Grande do Sul.

\section{REFERÊNCIAS BIBLIOGRÁFICAS}

CARDOSO, A. O. Relações entre a TSM nos oceanos Attântico e Pacífico e as condições climáticas nas regiões Sul e Sudeste do Brasil. Tese de Doutorado. ACA/IAG/ USP. São Paulo - SP, 158 pp. 2005.

CERA, J.C.; FERRAZ,S.E.T. Caracterização da Precipitação no Estado Do Rio Grande Do Sul. In: II Encontro Sul Brasileiro de Meteorologia, 2007, Anais... Florianópolis.

CERA, J.C.; FERRAZ,S.E.T.; BENDER,F.D. Influência da Oscilação Decadal do Pacífico e as Mudanças no Regime de Chuva do Rio Grande do Sul. In: VI Workshop Brasileiro de Micrometeorologia, 2009, Anais... Santa Maria, 2009.

DUCHON, C. E. Lanczos filtering in one and two dimensions. Journal of Applied Meteorology, v.18, p.1016-1022, 1979.

FERNANDES, V.; SPERLING, V.; MARQUES, J.R. Relação entre o Índice Oscilação Sul (IOS) e a Precipitação no Rio Grande do Sul. In: XVII Congresso de Iniciação Científica, 2009, Anais... Pelotas. XVIII Congresso de Iniciação Científica, 2009.

FERRAZ, S. E. T. Oscilações Intrasazonais no Sul e Sudeste do Brasil Durante o Verão. Tese (Mestrado em Meteorologia) - Universidade de São Paulo, São Paulo, 2000.

FERRAZ, S. E. T. Variabilidade Intrasazonal no Brasil e Sul da América do Sul. Tese (Doutorado em Meteorologia) Universidade de São Paulo, São Paulo, 2004.

HARE, S.R. Low frequency climate variability and salmon production. Tese (Ph.D.) School of Fisheries, University of Washington, Seattle, 1996.

HARMAN, H. H. Modern Factor Analysis. The University of Chicago Press, Chicago, IL, 1976.

JOLLIFFE, I.T.. Discarding Variables in a Principal Component Analysis. I: Artificial Data. Applied Statistics, 1972.

KAISER, HF: Computer-program for varimax rotation in factoranalysis. Educational and Psychological Measurement, v.19 (3), p. 413-420, 1959.

KAISER, HF: The varimax criterion for analytic rotation in factor-analysis Psychometrika, v. 23 (3), p. 187-200, 1958.

KIDSON, J. W. Principal Modes of Southern Hemisphere low- frequency variability obtained from NCEP- NCAR Reanalyses. Journal of Climate, v.12, p.2808- 2830, 1999.

LIEBMANN, B.; ALLURED, D. Daily precipitation grids for South America. Bulletin of the American Meteorological Society, 2005.
LORENZ, E.N. Empirical Orthogonal Functions and Statistical Weather Prediction, Sci. p 1. Statistical Forecasting Project, Department of Meteorology, MIT (NTIS AD 110268), 49 p. 1956.

MADDEN, R.A.; JULIAN, P.R. Detection of a 40-50 day oscillation in the zonal wind in the tropical Pacific. Journal of the Atmospheric Sciences 1971, v. 28, p.702-708

MADDEN, R.A.; JULIAN,P. R. Observations of the 40-50 day tropical oscillation - a review. Monthly Weather Review, v.122, p. 814-837, 1994

MANTUA, N.J.; HARE, S.R.; ZHAND, Y.; WALLACE, J.M.; FRANCIA, R.C. A Pacific Interdecadal Climate Oscillation With Impacts On Salmon Production: Bulletin of the American Meteorological Society, 78, 1069-1079, 1997.

MULAIK, S.A. The foundations of factor analysis. New York: McGraw-Hill Book Company. 1972.

NASCIMENTO, E. L. ; CALVETTI, L. . Identificação de condições precusoras de tempestades severas no sul do Brasil utilizando-se radiossondagens e parâmetros convectivos.. In: XIII Congresso Brasileiro de Meteorologia, 2004, Fortaleza. Anais... XIII Congresso Brasileiro de Meteorologia (CDROM). Fortaleza : Sociedade Brasileira de Meteorologia, 2004.

PAEGLE. J., N.; MO, K. C. Alternating wet and dry conditions over South America during summer. Monthly Weather Review, v.125, p.279-291, 1997.

PAEGLE, J. N.; BYERLE, L. A; MO, K. C. Intraseasonal modulation of South American summer precipitation. Monthly Weather Review, v. 128, p. 837-850, 2000.

PAMPUCH, L. A. Investigação do Modo Sul no Clima Presente e Futuro no Rio Grande do Sul. Dissertação (Mestrado em Meteorologia) - Universidade Federal de Santa Maria, Santa Maria, 2010.

RAO, V.B.; HADA, K. Characteristics of Rainfall over Brazil: Annual Variations and Connections with the Sourthern Oscillations. Theor. Appl.Climatol., v. 42, p. 81-91, 1990.

REBELLO, E. R. G. A Oscilação Decadal do Pacífico e sua Possível Influência no Regime de Precipitação do Rio Grande do Sul. In: XIV CBMET, 2006, Florianópolis - SC. Anais... XIV CBMET, 2006.

RECKZIEGEL, B. W. Levantamento dos Desastres Desencadeados por Eventos Naturais Adversos no Estado do Rio Grande do Sul no Período de 1980 a 2005. Tese (Mestrado em Geografia) - Universidade Federal de Santa Maria, Santa Maria, 2007.

RUMMEL RJ: Applied Factor Analysis, Northwestern University Press, Evanston, IL, 1970.

SINCLAIR, T.R.; LUDLOW, M.M. Influence of soil water supply on the plant water balance of four tropical grain legumes. Aust. J. Plant Physiol., 13:319-340, 1986. 
STRECK,N.A.; Martins, F. B.; Silva, J. C.;MORAIS, W. W.; SUSIN, F.; NAVROSKI, M. C.; VIVIAN, M. A. Deficiência hídrica no solo e seu efeito sobre transpiração, crescimento e desenvolvimento de mudas de duas espécies de eucalipto. Rev. Bras. Ciênc. Solo vol.32 no.3 Viçosa, 2008.

ZHANG, Y.; WALLACE,J. M.; BATTISTI, D. S. ENSO-like Interdecadal Variability, Journal of Climatology. 1997 v.10, p. $1000-1020$.
ZHANG, Y. An Observational Study of Atmosphere - Ocean Interaction in the Northern Oceans on Interannual and Interdecadal time- scales. Tese (PHD) - Universidade de Washington, Seattle 1996.

WILKS, D.S. Statistical Methods in the Atmospheric Sciences: an introduction. San Diego: Academic Press. 467 p. 1995. 